\title{
Unconventional UHF RFID Tags with Sensing and Computing Capabilities
}

\author{
Riccardo Colella, Danilo De Donno, Luciano Tarricone, and Luca Catarinucci
}

\begin{abstract}
The design of fully-passive UHF RFID tags preserving cost-effectiveness, yet supplying augmented capabilities, represents an ambitious and stimulating challenge, as such devices would pave the way to a large class of applications where identification, computation, automatic cognition, and wireless sensing are required. In this work, two solutions are proposed. The former, named RAMSES, is optimized for RFID-based sensing and relies on a novel approach exploiting a new-generation $I^{2} \mathrm{C}$-UHF RFID chip. RAMSES is able to write sensor data into the EPC and communicate up to 5 $m$ of distance from a conventional UHF RFID Class-1 Generation-2 (Gen2) reader. The latter solution, named SPARTACUS, renounces part of this long operating range in exchange for additional computing capabilities enabling an increased interaction with RFID readers. SPARTACUS represents the first example in literature of RFID device embedding sensing/actuation functionalities, distributed computation, and fully bidirectional communication with the reader. Satisfactory operating range, sensing, computation, data storage, and cost-effectiveness are the main strengths making the proposed devices definitely suitable for a wide array of novel and unconventional RFID applications.
\end{abstract}

Index Terms-RFID, tag, wireless sensor networks, computation, RF energy harvesting

\section{INTRODUCTION}

$\mathrm{T}$ HE emerging radio frequency identification (RFID) technology is more and more adopted in a huge range of application scenarios [1]-[5]. Nevertheless, in many appealing applications, a real added value would be given by the use of novel RFID devices which, while ensuring cost effectiveness and ease of use, also guarantee augmented functionalities, such as on-board sensing and computation.

The integration between RFID tags and sensors is a widely discussed topic in the literature. However, since complexity, size, and energy consumption are application-specific requirements of each sensor, the possibility to embed sensing functionalities into very small RFID chips is impracticable in most cases. On the contrary, compact fully-passive platforms allowing the RFID-based transmission of data gathered from on-board and/or external generic sensors is one of the alternative beaten paths. To the authors' knoeledge, the pioneers in developing augmented UHF RFID tags were Smith et al. in 2008 with their wireless identification and sensing

Manuscript received March 27, 2014; revised May 21, 2014.

Authors are with the Innovation Engineering Department, University of Salento, Via per Monteroni, 73100, Lecce, Italy.

Danilo De Donno is the corresponding author (e-mail: danilo.dedonno@ unisalento.it). platform (WISP) [6], which is the first battery-free programmable UHF RFID tag with sensors implementing the EPC Class-1 Generation-2 (Gen2 for short hereafter) protocol. A low-cost general-purpose alternative to the WISP is the sensor-tag (S-Tag) presented in the authors' earlier works [7] and shown in Fig. 1. Fabricated on a flexible substrate [8] and based on a multi-ID approach [9], the S-Tag can be connected to generic sensors and, when interrogated by a standard Gen2 reader, it is capable to transmit a proper combination of EPC codes that univocally encodes the sensor value. In [10] and [11], the electronic components of an augmented UHF RFID tag, including antenna, microcontroller unit (MCU), and sensors, are integrated in flexible organic substrates using inkjet-printing technology. Moreover, the authors address the integration of carbon-nanotubes on paper substrates for the fabrication of ultra-sensitive gas sensors and present benchmarking results for different scavenging approaches involving solar and charge transfer-based mechanisms. A passive multi-standard RFID tag enhanced with sensing and localization functionalities and implemented in a $0.13-\mu \mathrm{m}$ bulk CMOS process is presented in [12]. An interesting design strategy for fully-passive RFID sensors is proposed in [13][16]. It relies on detecting variations in gain, input impedance, and differential radar cross-section of the tag's antenna caused by environmental changes (e.g. temperature [13] and humidity [14]) or mechanical stresses (e.g. strain [15] and motion [16]). This sensing mechanism, besides being extremely susceptible to radio propagation phenomena, is not compatible with existing RFID infrastructures since it requires expensive equipment, such as vector network analyzers or customized

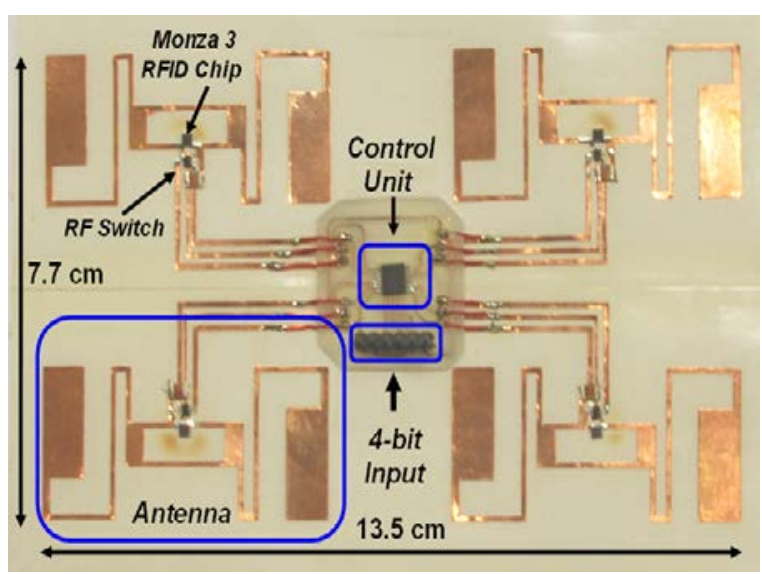

Fig. 1. The Sensor Tag (S-Tag) prototype.

receivers, to reliably extract sensor-dependent characteristics from backscattered radio signals. 


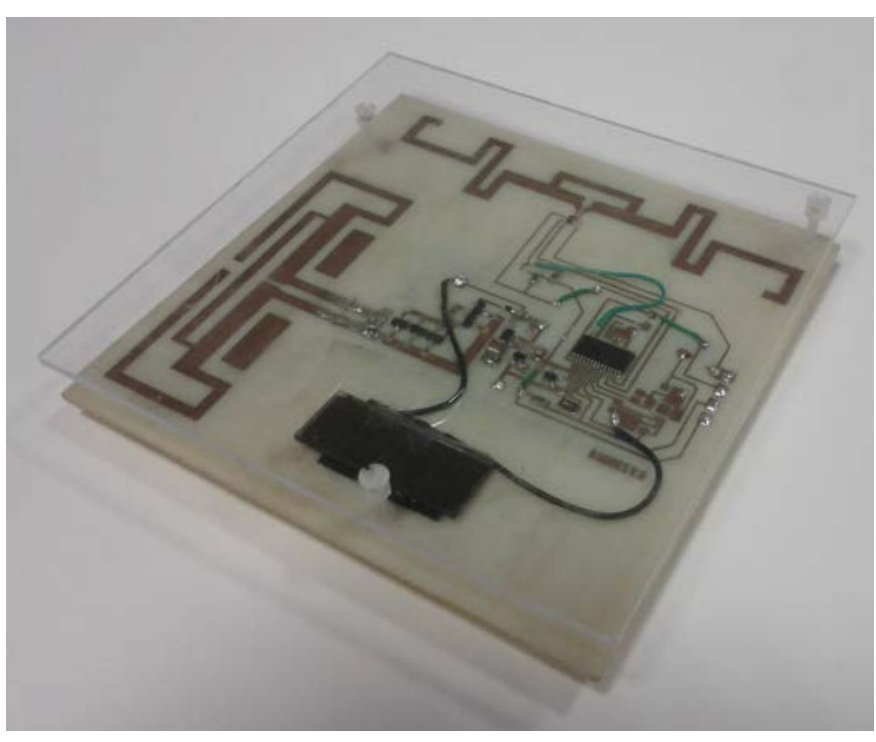

Fig. 2. Preliminary RAMSES prototype (dimensions $9.5 \times 9.5 \times 1.6 \mathrm{~cm}^{3}$ ).

In addition to the academic research, RFID manufacturers are starting to commercialize Gen2 tags that incorporate sensing, computation, and data-logging capabilities for unconventional RFID applications. Among them, it is worth mentioning the SL900A sensory tag by Austria Micro Systems (AMS), the Easy2Log tag by CAEN RFID, and the SensTAG by Phase IV. Nevertheless, none of the aforementioned solutions comprises all the primary characteristics required by future RFID-based sensing applications, e.g. the full compliance with RFID standards and regulations (most devices need specific settings for the reader), a satisfactory operating range (comparable to that of conventional UHF passive tags), a variety of on-board sensors, high expansibility and programmability.

In this work, two smart RFID devices representing a substantial step ahead of the S-Tag are introduced. The first device, named RAMSES (RFID Augmented Module for Smart Environmental Sensing), is a fully-passive platform optimized for long-range RFID sensing. Equipped with on-board sensors, interfaces for generic external sensors, a new-generation $\mathrm{I}^{2} \mathrm{C}$ UHF RFID chip, and a high-performance energy-harvesting circuit, RAMSES is able to dynamically update its EPC code with actual sensor measurements and communicate up to $5 \mathrm{~m}$ of distance from a conventional Gen2 reader. The second device, named SPARTACUS (Self-Powered Augmented RFID Tag for Autonomous Computation and Ubiquitous Sensing), provides different capabilities compared to RAMSES. Indeed, in spite of a shorter operating range, SPARTACUS embeds sensing and actuating functionalities, distributed computation, and fully bidirectional communication with Gen2 readers. Moreover, it is able to perform simple computations on the basis of recorded events, thus paving the way to new classes of applications, as envisioned at the end of the paper.

\section{RAMSES}

RAMSES is a long-range, Gen2-compliant, and programmable RFID Augmented Module for Smart Environmental Sensing. It relies on a novel approach

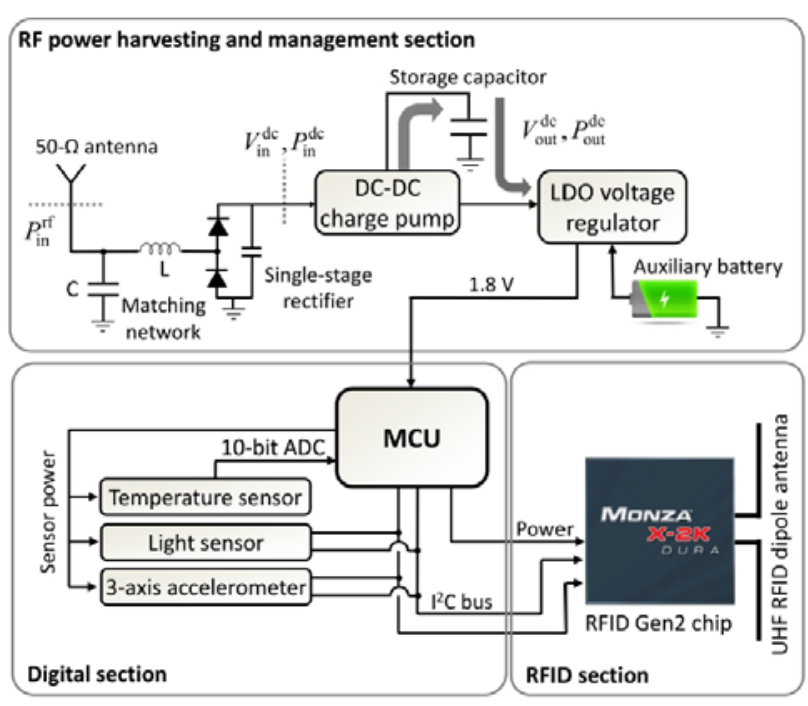

(a) RAMSES architecture

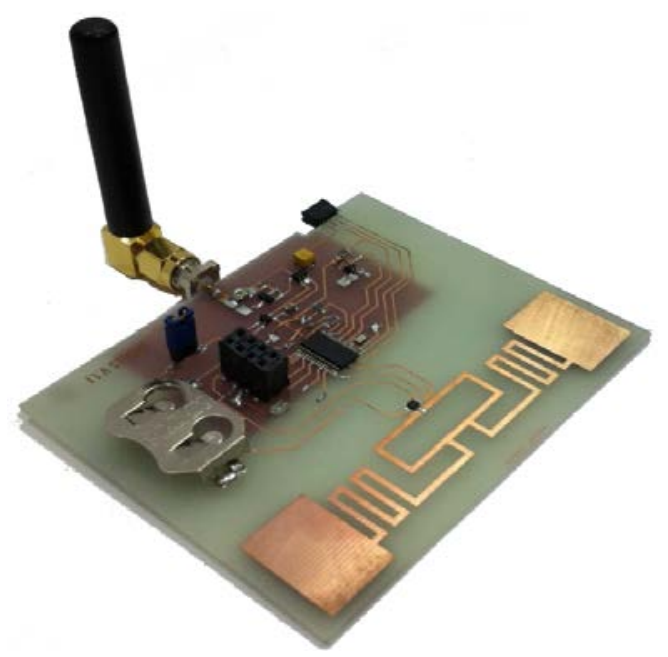

(b) RAMSES prototype photo

Fig. 3. Architecture (a) and prototype photo (b) of the last RAMSES revision (dimensions 8x8 $8 \mathrm{~cm}^{3}$ ).

exploiting a new-generation RFID chip with dual communication interface: a wired $\mathrm{I}^{2} \mathrm{C}$ interface managed by a microcontroller and a wireless UHF interface for communication with standard RFID Gen2 readers. A preliminary RAMSES prototype (see Fig. 2) has been presented in our prior work [17] and a lot of work has been done in the last year to improve the whole architecture, to optimize the design, and to enhance significantly both performance and functionalities. More specifically, the main advances concern the harvester design [18] which provides the current version of RAMSES with more than double communication range. Secondly, in addition to the sole temperature sensor of the preliminary version, RAMSES is now equipped with light and acceleration sensors. Lastly, compared to the RAMSES revision presented in [19], the BAP (Battery Assisted Passive) mode has been optimized and improved mainly in terms of data-logging capabilities. 


\section{A. Design and implementation}

A block diagram of the RAMSES architecture is depicted in Fig. 3(a) while a prototype photo of the last RAMSES revision is shown in Fig. 3(b). The operating principle is simple yet effective. A conventional UHF RFID tag, composed of a dipole-like antenna and a Gen2 chip, makes up the RFID section. The main feature of the adopted RFID chip is the capability of its memory to be accessed via the wired $\mathrm{I}^{2} \mathrm{C}$ interface (in addition to the standard Gen2 air interface). Consequently, sensor data transferred over the $\mathrm{I}^{2} \mathrm{C}$ bus by means of an MCU are directly accessible to standard RFID Gen2 readers. The power needed to operate RAMSES could be definitely retrieved by a battery (BAP mode), but also harvested from the RFID interrogation signal emitted by the reader. To this end, RAMSES is equipped with an RF power harvesting and management section comprising a $50-\Omega$ whip antenna matched to a single-stage full-wave RF-DC converter. The Seiko Instruments S-882Z24 charge pump IC adopted to step-up the rectified voltage. Such a DC-DC converter implements fully-depleted SOI technology to enable ultra-lowvoltage operation. In fact, when its input voltage is $0.35 \mathrm{~V}$ or higher the oscillation circuit starts operating and the steppedup electric power is accumulated in a storage capacitor. When the capacitor reaches $2.4 \mathrm{~V}$, the integrated supervisory circuit of the S-882Z24 automatically releases the stored energy to a 1.8-V low-dropout (LDO) voltage regulator which power up the digital section. When the voltage on the storage capacitor decreases to approximately $1.85 \mathrm{~V}$ as a result of its discharge, the S-882Z24 disconnects its output and starts a new charging process. In the BAP case, the RF energy-harvesting module is bypassed since the $3-\mathrm{V}$ lithium cell provides entirely the power needed to operate RAMSES.

RAMSES is equipped with a 16-bit TI MSP430F5132 ultralow-power MCU running up to $12 \mathrm{MHz}$ with $1.8-\mathrm{V}$ supply voltage (180- $\mu \mathrm{A} / \mathrm{MHz}$ supply current) and providing $8 \mathrm{kB}$ of flash memory, $1 \mathrm{kB}$ of SRAM, and eight 10-bit 200-ksps Analog-to-Digital Converter (ADC) channels. The MCU is programmed with an energy-efficient firmware running at 1 $\mathrm{MHz}$ and implementing $\mathrm{I}^{2} \mathrm{C}$ and ADC sampling routines. The 10-bit ADC samples a TI LM94021 analog temperature sensor consuming down to $9 \mu \mathrm{A}$. Then, readings from an ADXL346 accelerometer and a MAX44009 ambient light sensor are taken via the $\mathrm{I}^{2} \mathrm{C}$ interface. The ADXL346 is an ultra-low power (90- $\mu$ A current consumption) 3-axis accelerometer with high-resolution measurements (13 bits/axis). The MAX44009, instead, is the industry's lowest-power ambient light sensor (1 $\mu \mathrm{A}$ current consumption) with 16-bit resolution. The sensor readings are organized and written into the EPC memory banks of the RFID Gen2 chip. Once interrogated, the EPC frames are delivered to the reader using zero-power backscatter modulation.

\section{B. Experiments}

RAMSES has been fabricated in our labs by using a photolithography process on FR4 substrate and handy soldering off-the-shelf discrete components. The RFID antenna is patterned directly on the PCB while an SMA connector allows to connect generic $50-\Omega$ UHF antennas to the harvester. A small female header exposes the $\mathrm{I}^{2} \mathrm{C}$ bus and

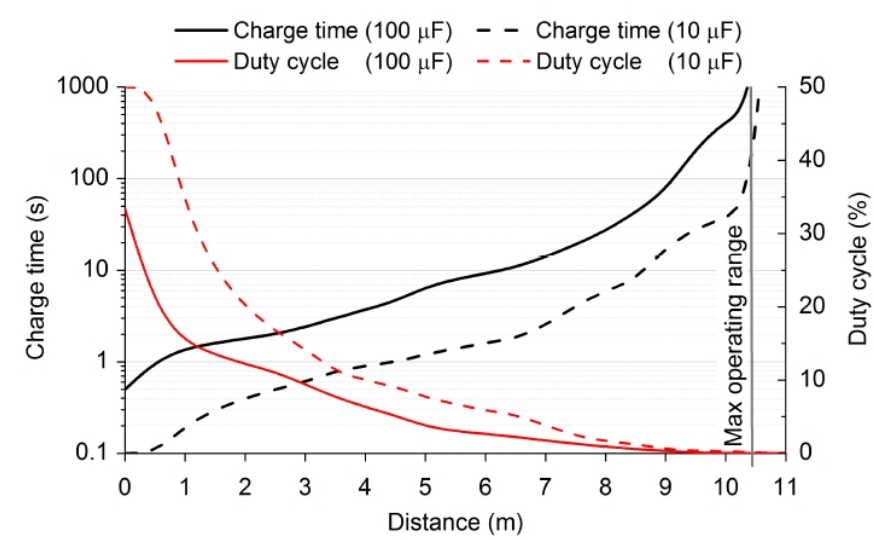

Fig. 4. Measured average time needed for the harvester to charge $10 \mu \mathrm{F}$ and $100 \mu \mathrm{F}$ storage capacitors (primary y axis) and achieved duty cycle (secondary y axis) when varying the RAMSES-reader distance.

other MCU ports for future expansions to external sensors and devices. In order to analyze the RAMSES behavior and evaluate its performance and sensing capabilities in real operating conditions, a series of experiments has been carried out.

As previously outlined, the power absorbed by MCU, sensors, and $\mathrm{I}^{2} \mathrm{C}$-RFID chip causes the storage capacitor to discharge. More specifically, when the voltage on the storage capacitor declines approximately to $1.85 \mathrm{~V}$, the charge-pump IC automatically stops the discharge process by disconnecting its output. The idle time between MCU operations, i.e. the duty cycle of the overall system, is determined by the amount of input power to the charge-pump IC and by the size of the storage capacitor. In fact, since for a given task the MCU execution time $T_{\text {on }}$ is fixed, RAMSES duty cycle:

$$
\text { Duty cycle }=\frac{T_{\text {on }}}{T_{\text {on }}+T_{\text {charge }}}
$$

can be maximized by minimizing the time $T_{\text {charge }}$ needed by the harvester to charge up the storage capacitor. The time $T_{\text {on }}$ needed for the MCU to complete its tasks (i.e. to take measurements from three sensors and communicate with the RFID chip via the $\mathrm{I}^{2} \mathrm{C}$ interface) has been experimentally found to be approximately $250 \mathrm{~ms}$.

In order to evaluate RAMSES performance in terms of storage capacitor charge time and duty cycle, a commercial RFID Gen2 reader set with the maximum allowable transmit power for the European regulations, i.e. 3.2-W EIRP in the 865-868 MHz frequency band, has been used to energize and interrogate RAMSES at different distances. The experiments have been conducted in a large lecture room with reader antenna and RAMSES placed in the line of sight (LOS) $1.5 \mathrm{~m}$ above the floor, both oriented in the maximum-gain direction. As shown in Fig. 4, RAMSES is able to autonomously operate (fully-passive mode) up to approximately $10 \mathrm{~m}$ of distance from the reader. Since the rapidity of the DC-DC charge pump to build up the output voltage depends on the amount of RF energy incident on the harvesting antenna, the time needed to charge the storage capacitor increases with the distance. Obviously, a smaller capacitor takes less time to be charged at the cost of a reduced number of tasks and functionalities 


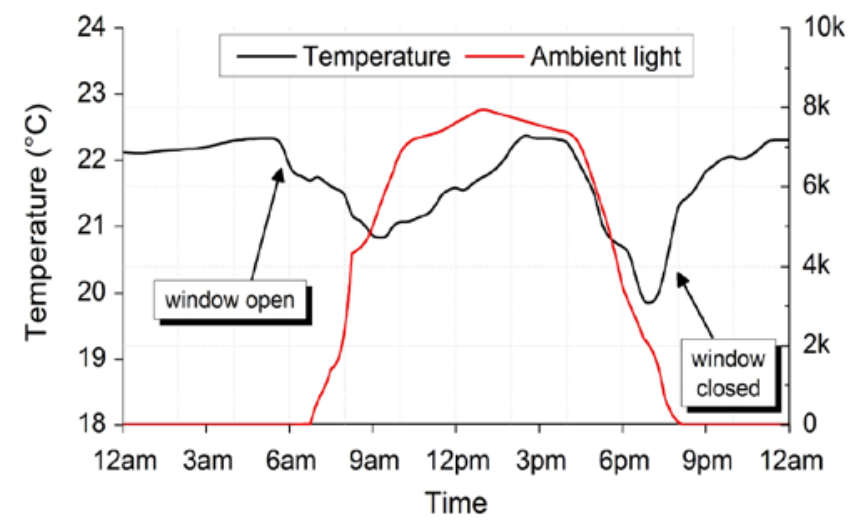

(a) Environmental sensing performed by RAMSES

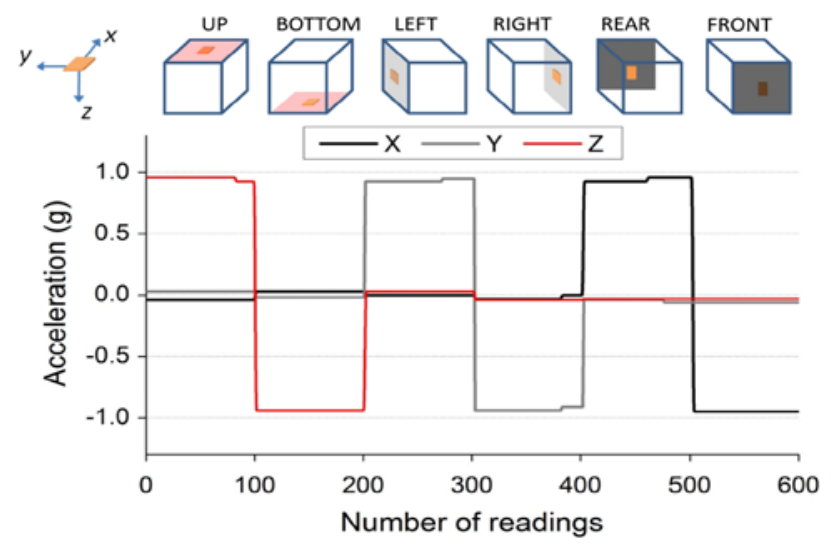

(b) Static acceleration measurements performed by RAMSES

Fig. 5. 24-hour temperature and light monitoring in an indoor scenario (a) and static acceleration measurements performed by RAMSES attached to an internal face of a parcel (b).

runnable by the MCU. It has been found experimentally that a $10 \mu \mathrm{F}$ capacitor is sufficient to perform only temperature measurements while the full RAMSES functionalities (temperature, light, and acceleration measurements) are feasible with a $100 \mu \mathrm{F}$ capacitor. More experimental results performed by a customized Software Defined Radio (SDR) testing platform [20], [21] are available from [19].

A series of tests aimed at verifying RAMSES capabilities to perform environmental sensing has been conducted in realworld application scenarios (see Fig. 5). In the former, RAMSES has been used to monitor ambient temperature and light conditions over a 24-hour observation period. In the latter, static acceleration measurements on a parcel have been logged by RAMSES in BAP mode in order to verify its ability to catch abusive handling events during a shipment. This last experiment has also demonstrated the ability of RAMSES to perform RFID-based sensor data transmissions up to approximately $22 \mathrm{~m}$ of distance from the interrogator in BAP mode. To the best of the authors' knowledge, this represents the longest communication distance ever reported for similar sensor-enhanced RFID devices.

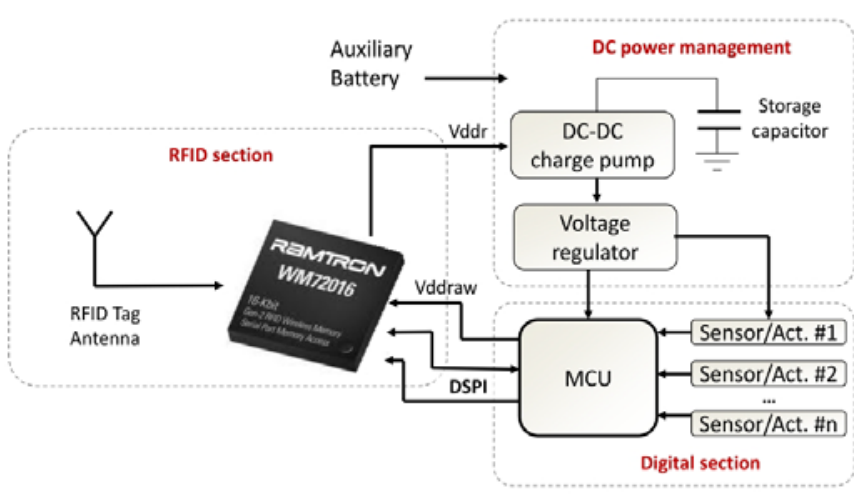

Fig. 6. SPARTACUS architecture.

\section{SPARTACUS}

SPARTACUS is an augmented RFID tag conceived for complex applications requiring the local computation and storage of data coming from heterogeneous sensors along with the control of actuators.

\section{A. Design and implementation}

The block diagram of SPARTACUS architecture, slightly different from the RAMSES one, is reported in Fig. 6. More specifically, the goal is not here to maximize the RFID sensing range, but on the contrary to realize an RFID device capable to sense, elaborate, transmit data towards a reader and, in the meanwhile, capable to receive commands from the reader itself, to change the elaboration status and, if required, to control actuators.

The core of SPARTACUS is the RAMTRON WM72016 RFID chip featuring a 16-Kb F-RAM memory and integrating both an RFID Gen2-compliant wireless interface working in UHF band and a wired DSPI port for read/write operations. It is worth highlighting that F-RAM technology provides 30 times larger and a 6 times faster memory if compared to conventional EEPROMs with obvious advantages in terms of time and power efficiencies. The control unit of SPARTACUS is realized with the Microchip PIC18F46J11 MCU. The MCU on the one hand drives internal or external sensors/actuators, on the other hand controls the communication with the WM72016 memory via DSPI port. Both memory and microcontroller are optimized for low-power operations. Data, commands, and interrupts may be continuously and mutually exchanged between the reader and SPARTACUS in a symmetrical, bidirectional way. Indeed, the identical read and write sensitivities of the WM72016 chip enable read and write operations at exactly the same distance. The main advantage is that, whenever a reader should be able to read data from SPARTACUS, it could also send back new instructions or controls.

\section{B. Experiments}

A preliminary SPARTACUS prototype is shown in Fig. 7. It comprises antenna, power management section, MCU, photo-resistor (as an example of analog light sensor), LED (emulating an actuator), and slot for auxiliary battery. As shown, SPARTACUS antenna exploits meander lines to 


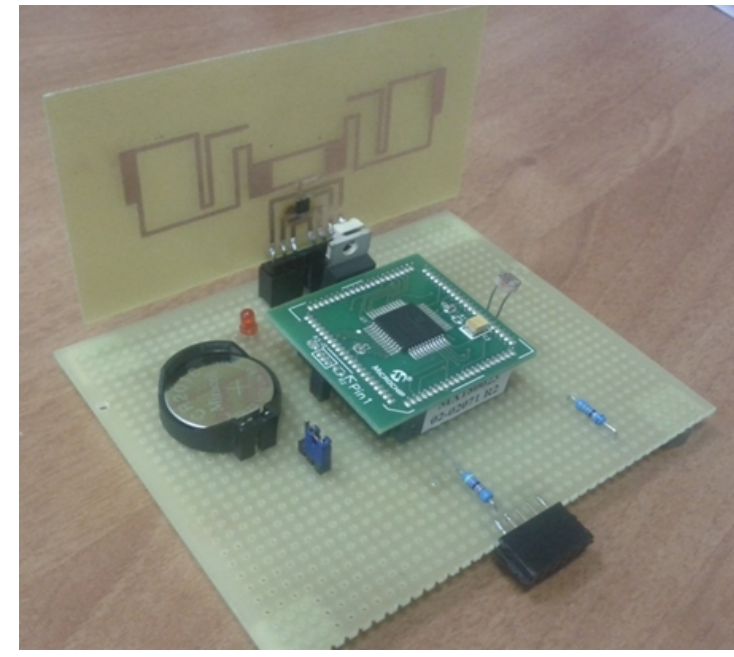

Fig. 7. Preliminary SPARTACUS prototype.

achieve a very compact form factor $\left(7.1 \times 1.9 \mathrm{~cm}^{2}\right)$. Detailed simulations have been performed in CST Microwave Studio by setting the minimization of the reflection coefficient at the desired frequency (i.e. $866.5 \mathrm{MHz}$ ) as the fitness function and by adopting a gradient-based interpolated quasi-newton optimizer. As shown in Fig. 8(a), the simulated -19.6-dB reflection coefficient magnitude around $866.5 \mathrm{MHz}$ demonstrates the good impedance matching between antenna and RFID chip (63-j199 $\Omega$ is the complex impedance of the chip). The E-plane polar diagram in Fig. 8(b) depicts the typical dipole radiation pattern (1.9 dBi is the realized gain) achieved, via simulations, for the SPARTACUS antenna. Note that, depending on the application, a directive patch antenna could be used to achieve higher performance (e.g. a longer read range) and platform tolerance [22]-[24].

The considerable amount of memory, along with the energetic autonomy, and the wireless fully bidirectional communication, enable a series of new applications where sensor data have to be shared with the reader (rather than stored in the memory) or locally processed to control actuators and generate alerts. As a simple proof of concept, a test highlighting the aforementioned SPARTACUS capabilities has been carried out. An auxiliary battery is necessary in this case since a LED, simulating a real actuator, need to be driven. The arranged setup for the demo is shown in Fig. 9. SPARTACUS has been programmed to turn on the LED - or equivalently to activate an actuator - when the environmental light level detected by the local sensor is lower than a preset threshold - dashed green line at the bottom of Fig. 10(a). On the other side, an Impinj Speedway Revolution reader has been connected to a PC and a DEMO application capable to process light data transmitted by SPARTACUS and send back instructions has been implemented. In this specific case, the task of the reader concerns simply the turning off of the LED (actuator) only when the received light level exceeds a certain threshold - dashed red line at the top of Fig. 10(a). The test has been performed in a room of approximately $15 \mathrm{~m}^{2}$, by moving SPARTACUS in the reader coverage area, and by varying the ambient light intensity for a total test time of 300 s. In Fig. 10a, the blue continuous line is referred to the light

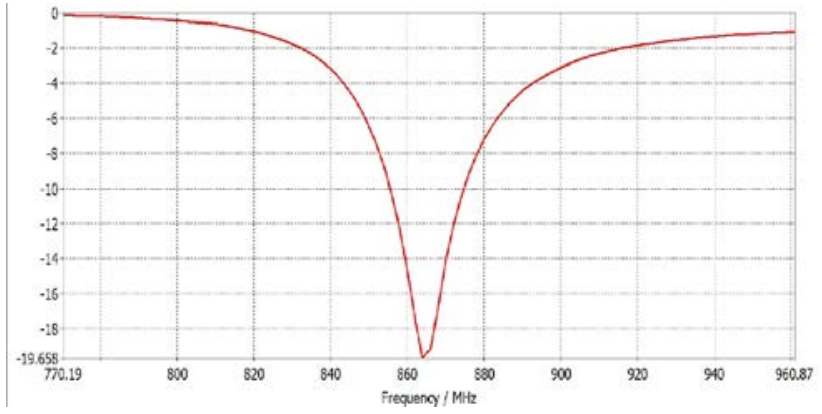

(a) Magnitude of the reflection coefficient

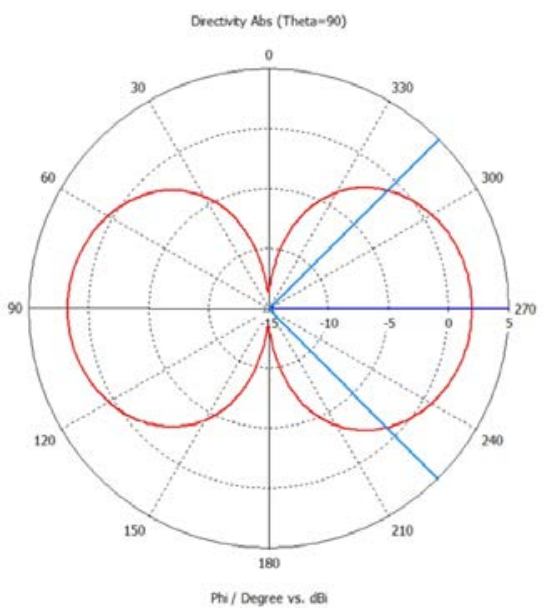

(b) E-plane polar radiation diagram

Fig. 8. SPARTACUS antenna simulations performed by CST MW Studio.

values transmitted by SPARTACUS during the test. This demonstrates the effectiveness of SPARTACUS to sense and transmit sensor data towards a traditional RFID reader. Moreover, as shown in Fig. 10(b), the LED is correctly activated (by SPARTACUS) and deactivated (by the RFID reader), according to preset thresholds. Specifically, continuous green spikes represent the LED-activation events locally performed by SPARTACUS, whilst dashed red spikes represent LED-deactivation commands logically computed by the reader and communicated to SPARTACUS.

Although extremely simple, this test clearly demonstrates that, differently from canonical RFID devices, SPARTACUS enables a real distributed computation between the reader and the tag. Thanks to this characteristic, it is expected that several SPARTACUS nodes powered by different RFID readers could cooperatively interact and change their behavior depending on stored data, events, actions to undertake. This approach represents a suitable way to implement, de facto, newgeneration heterogeneous and self-organizing sensor networks [25].

\section{CONCLUSION}

Two different computational RFID devices with augmented capabilities have been introduced in this paper. The first one, named RAMSES, is a robust sensing platform equipped with the $I^{2} \mathrm{C}$-UHF RFID Monza X-2K chip by Impinj, an efficient energy-harvesting system, an ultra-low power microcontroller, and on-board sensors. Sensor data, stored into the EPC memory banks of the RFID chip, can be rapidly accessed and 

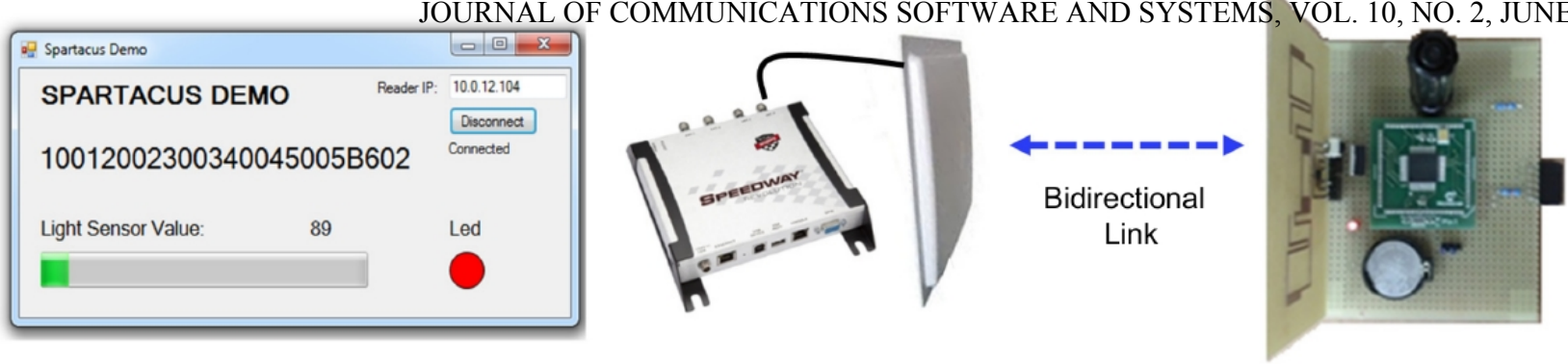

Fig. 9. The SPARTACUS demo running on a general-purpose PC connected to the Impinj Speedway Revolution RFID reader.

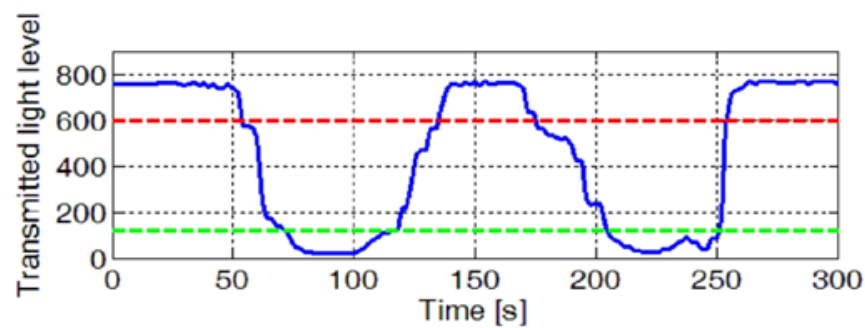

(a)

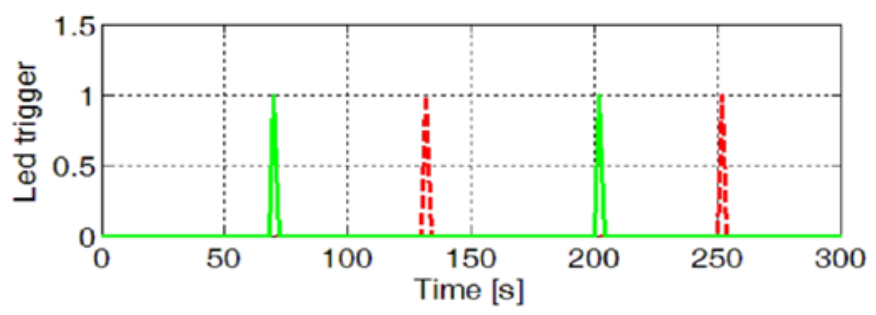

(b)

Fig. 10. Light level transmitted by SPARTACUS (a) and LED control trigger events (b).

managed by conventional Class- 1 Generation-2 readers. The second device, named SPARTACUS, is similar to RAMSES but exhibits some peculiarities, such as bidirectional memory access, larger memory size, and compact dimensions, which pave the way to new classes of applications requiring on-board processing of sensor data. Features and differences of both platforms make them definitely suitable for different use cases, on the basis of user-specific needs and performance requirements.

\section{REFERENCES}

[1] L. Catarinucci, R. Colella, M. De Blasi, V. Mighali, L. Patrono, and L. Tarricone: High Performance RFID Tags for Item-Level Tracing Systems, Proceedings of SoftCOM 2010 - International Conference on Software, Telecommunications and Computer Networks, pp. 21-26, 2010.

[2] G. Calcagnini, F. Censi, M. Maffia, L. Mainetti, E. Mattei, L. Patrono, and E. Urso: Evaluation of Thermal and Nonthermal Effects of UHF RFID Exposure on Biological Drugs, IEEE Transactions on Information Technology in Biomedicine, vol. 16, no. 6, pp. 1051-1057, 2012.

[3] M. Maffia, L. Mainetti, L. Patrono, and E. Urso: Evaluation of Potential Effects of RFID-based Item-Level Tracing Systems on the Integrity of Biological Pharmaceutical Products, International Journal of RF Technologies: Research and Applications, vol. 3, no. 2, pp. 101-118, 2012.

[4] D. Alessandrelli, L. Mainetti, L. Patrono, G. Pellerano, M. Petracca, and M. L. Stefanizzi: Implementation and Validation of an Energy-Efficient MAC Scheduler for WSNs by a Test Bed Approach, $201220^{\text {th }}$ International Conference on Software, Telecommunications and Computer Networks (SoftCOM 2012), 2012.

[5] L. Catarinucci, R. Colella, L. Mainetti, L. Patrono, S. Pieretti, I. Sergi, and L. Tarricone: Smart RFID Antenna System for Indoor Tracking and
Behavior Analysis of Small Animals in Colony Cages, IEEE Sensors Journal, vol. 14, no. 4, pp. 1198-1206, Apr. 2014.

[6] A. P. Sample, D. J. Yeager, P. S. Powledge, A. V. Mamishev, and J. R. Smith: Design of an RFID-based Battery-Free Programmable Sensing Platform, IEEE Transactions on Instrumentation and Measurement, vol. 57, no. 11, pp. 2608-2615, Nov. 2008.

[7] L. Catarinucci, R. Colella, L. Tarricone: Enhanced UHF RFID SensorTag, IEEE Microwave and Wireless Components Letters, vol. 23, no. 1, pp. 49-51, 2013.

[8] L. Catarinucci, R. Colella, and L. Tarricone: Smart Prototyping Techniques for UHF RFID Tags: Electromagnetic Characterization and Comparison with Traditional Approaches, Progress In Electromagnetics Research, vol. 132, pp. 91-111, 2012.

[9] L. Catarinucci, R. Colella, and L. Tarricone: Sensor Data Transmission Through Passive RFID Tags to Feed Wireless Sensor Networks, 2010 IEEE MTT-S International Microwave Symposium Digest (IMS 2010), 2010.

[10] L. Yang, A. Rida, R. Vyas, and M. M. Tentzeris: RFID Tag and RF Structures on a Paper Substrate Using Inkjet-Printing Technology, IEEE Transactions on Microwave Theory and Techniques, vol. 55, no. 12, Dec. 2007.

[11] R. Vyas, V. Lakafosis, H. Lee, G. Shaker, L. Yang, G. Orecchini, A. Traille, M. M. Tentzeris, and L. Roselli: Inkjet Printed, Self Powered, Wireless Sensors for Environmental, Gas, and Authentication-based Sensing, IEEE Sensors Journal, vol. 11, no. 12, pp. 3139-3152, Dec. 2011.

[12] T. Ussmueller, D. Brenk, J. Essel, J. Heidrich, G. Fischer, and R. Weigel: A Multistandard HF/UHF-RFID-tag with Integrated Sensor Interface and Localization Capability, 2012 IEEE International Conference on RFID, pp. 66-73, April 2012.

[13] S. Capdevila, L. Jofre, J. Romeu, and J. C. Bolomey: Multi-Loaded Modulated Scatterer Technique for Sensing Applications, IEEE Transactions on Instrumentation and Measurement, vol. 62, no. 4, pp. 794-805, Apr. 2013.

[14] J. Virtanen, L. Ukkonen, T. Björninen, A. Z. Elsherbeni, and L. Sydanheimo: Inkjet-Printed Humidity Sensor for Passive UHF RFID Systems, IEEE Transactions on Instrumentation and Measurement, vol. 60, no. 8, pp. 2768-2777, Aug. 2011.

[15] C. Occhiuzzi, C. Paggi, and G. Marrocco: Passive RFID Strain-Sensor Based on Meander-Line Antennas, IEEE Transactions on Antennas and Propagation, vol. 59, no. 12, pp. 4836-4840, Dec. 2011.

[16] C. Occhiuzzi, S. Cippitelli, and G. Marrocco: Modeling, Design and Experimentation of Wearable RFID Sensor Tag, IEEE Transactions on Antennas and Propagation, vol. 58, no. 8, pp. 2490-2498, Aug. 2010.

[17] D. De Donno, L. Catarinucci, and L. Tarricone: Enabling Self-Powered Autonomous Wireless Sensors with New-Generation $I^{2} C$-RFID Chips, 2013 IEEE MTT-S International Microwave Symposium Digest, pp. 14, June 2013.

[18] D. De Donno, L. Catarinucci, and L. Tarricone: An UHF RFID EnergyHarvesting System Enhanced by a DC-DC Charge Pump in Silicon-onInsulator Technology, IEEE Microwave and Wireless Components Letters, vol. 23, no. 6, June 2013.

[19] D. De Donno, L. Catarinucci, and L. Tarricone: RAMSES: RFID Augmented Module for Smart Environmental Sensing, IEEE Transactions on Instrumentation and Measurement, vol. 63, no. 7, July 2014.

[20] D. De Donno, L. Catarinucci, R. Colella, F. Ricciato, and L. Tarricone: Differential RCS and Sensitivity Calculation of RFID Tags with Software-Defined Radio, 2012 IEEE Radio and Wireless Symposium (RWS 2012), 2012.

[21] D. De Donno, L. Tarricone, L. Catarinucci, V. Lakafosis, and M. M. Tentzeris: Performance Enhancement of the RFID EPC Gen2 Protocol 
by Exploiting Collision Recovery, Progress in Electromagnetics Research B, vol. 43, pp. 53-72, 2012.

[22] L. Catarinucci, S. Tedesco, D. De Donno, and L. Tarricone: PlatformRobust Passive UHF RFID Tags: A Case-Study in Robotics, Progress in Electromagnetics Research C, vol. 30, pp. 27-39, 2012.

[23] H. Lee, S. Kim, D. De Donno, and M. M. Tentzeris: A Novel Universal Inkjet-Printed EBG-backed Flexible RFID for Rugged On-Body and Metal Mounted Applications, 2012 IEEE MTT-S International Microwave Symposium Digest, pp. 1-4, 2012.

[24] L. Catarinucci, R. Colella, and L. Tarricone: Design, Development and Performance Evaluation of a Compact and Long-Range passive UHF RFID Tag, Microwave and Optical Technology Letters, vol. 54, no. 5, pp. 1335-1339, May 2012.

[25] A. Capone, M. Cesana, D. De Donno, and I. Filippini: Optimal Placement of Multiple Interconnected Gateways in Heterogeneous Wireless Sensor Networks, NETWORKING 2009, Lecture Notes in Computer Science (LNCS), vol. 5550, pp. 442-455, 2009.

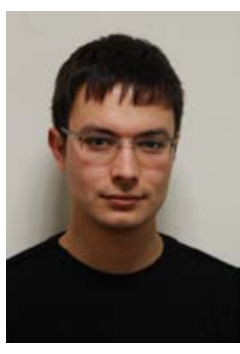

Riccardo Colella is a Ph.D Student at the University of Salento, Lecce, Italy. He received the M.Sc. degree with honors in Telecommunication Engineering from the University of Salento in 2010. Since September 2007, he has been collaborating with the Electromagnetic Fields Group (EML2) at the University of Salento. His research activity is focused on the design and optimization of RFID sensor-tags and their applications in healthcare and in wireless sensor networks (WSNs). He has authored more than 30 publications on international journals and conferences and two chapter books with international diffusion. He is a holder of a patent.

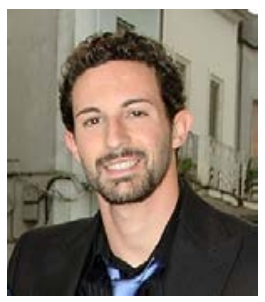

Danilo De Donno was born in Lecce, Italy, in 1983. He received the B.Sc. and M.Sc. degrees (cum laude) in telecommunication engineering from Politecnico di Milano, Italy, in 2005 and 2008, respectively, and the Ph.D. degree in information engineering from the University of Salento, Lecce, in 2012. In 2011, he was a Visiting Researcher with the School of Electrical and Computer Engineering, Georgia Institute of Technology, Atlanta, GA, USA. He is currently a PostDoctoral Fellow with the Innovation Engineering Department, University of Salento. His research interests include the design of parallel algorithms on graphics processors, computational RFID systems, and software-defined radio experimentation.

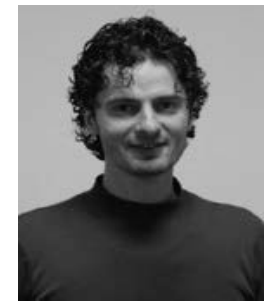

Luca Catarinucci is an Assistant Professor in Electromagnetic Fields at the Department of Innovation Engineering, University of Salento, Italy. His research activity is mostly focused on the implementation of electromagnetic simulation tools, in the FDTD analysis of human-antenna interaction and in the electromagnetic characterisation of materials. Further contributions deal with the time domain reflectometry (TDR) for the characterisation of fluids and in the radiofrequency identification (RFID) antenna and system design. He authored more than 50 papers on international and national journals and in international and national conferences and a chapter of a book with international diffusion.

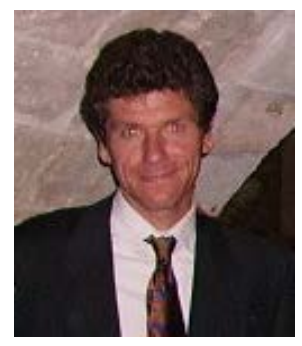

Luciano Tarricone received the Laurea degree (cum laude) in electronic engineering and the Ph.D. degree from Rome University La Sapienza, Rome, Italy, in 1989 and 1994, respectively. Since 1994, he has been a Researcher with the University of Perugia, Italy, and since 1998, he has been a Professore Incaricato of EM fields and EM compatibility. Since 2001, he has been a Faculty Member with the Department of Innovation Engineering, University of Salento, Lecce, Italy, where he is a Full Professor of electromagnetic fields. He has authored over 300 scientific papers. His main contributions are in the modelling of microscopic interactions of EM fields and biosystems, and in numerical methods for efficient computeraided design (CAD) of microwave circuits and antennas. He is currently involved in bioelectromagnetics, EM energy harvesting and wireless power transmission, novel CAD tools and procedures for MW circuits, RFID, and EM highperformance computing. 